\title{
Ear Modeling and Sound Signal Processing
}

\author{
Jack Xin *
}

\begin{abstract}
Ear modeling can significantly improve sound signal processing and the design of hearing devices. Ear models based on mechanics and neural phenomenology of the inner ear (cochlea) form a class of nonlinear nonlocal dispersive partial differential equations (PDEs). These PDEs capture the essential nonlinear phenomena in hearing, such as combination frequency generation, compression, and suppression, in agreement with experimental data. The PDEs help to generate linear and nonlinear auditory transforms, including a class of orthogonal discrete auditory transforms with broader sprectrum than that of the discrete Fourier transform, consistent with human audition. The PDEs also facilitates an alternative sound amplification method for hearing aid design.
\end{abstract}

${ }^{*}$ Department of Mathematics and ICES, University of Texas at Austin, Austin, TX 78712. Email: jxin@math.utexas.edu. Partially supported by NSF-ITR 0219004 and a Guggenheim fellowship. 


\section{Introduction}

Mathematical models of human auditory systems can contribute tremendously to the understanding of experimental hearing data and sound signal processing. A success story is MP3, a remarkable sound compression tool that employed empirical models of hearing threshold curves of human sound perception to significantly reduce the bit width of digital sounds $[5,31]$. It is routinely used for downloading and playing digital music.

Scientifically, it is important to develop a systematic framework of ear models based on available hearing data, and tone them up into new tools of signal processing. Even though human auditory systems (or those of mammals such as cat, chinchila, squirrel monkey) are complex multi-scale dynamical systems, much progess has been made in modeling the key parts of auditory information processing. As sound waves enter our ears, the first stage of signal processing occurs on basilar membrane (BM) of the inner ear (cochlea). Sound waves of different frequencies induce vibrations at distinct locations on BM. The frequency and location correspondence is like that of a piano keyboard. However, there is more to it in the cochlea. The BM is connected with sensory hair cells and peripheral nerve fibers. The next level of information processing occurs in as many as 30,000 neural fibers from the inner ear to the brain. Nonlinearities are associated with peripheral auditory neurons when the hair cells convert sound signals from mechanical to neural representation. It is now well known [11] that outer hair cells (OHCs) of the cochlea play an active role in increasing the sensitivity and dynamic range of ear. The frequency distribution of sound is maintained in the wave patterns on the BM, and is preserved along the fibers, resulting in tonotopic organization of frequency responses in the auditory cortex of the brain. The last level of the information flow is sound perception. The study of the relation between sounds and their perception is psychoacoustics, where enormous data have been collected from human subjects. Interestingly, as sound information reaches higher levels, many nonlinear features remain. 

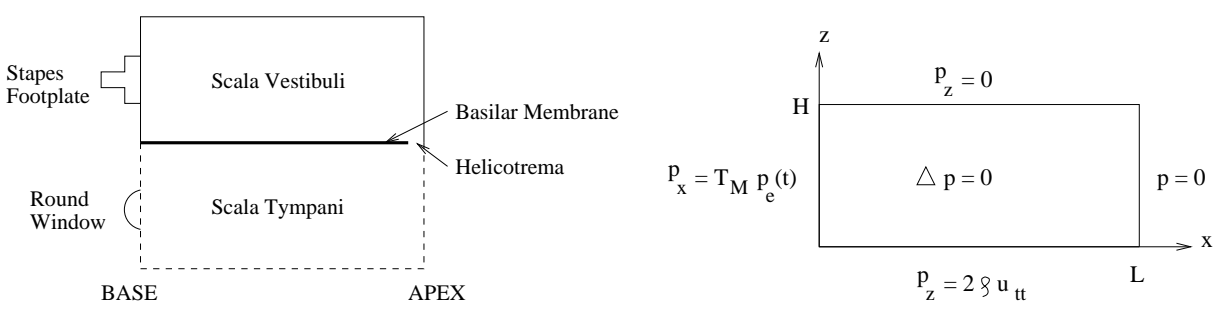

Figure 1: The left figure is a schematic of the cochlea chambers filled with viscous fluid. The right figure shows the upper chamber with the fluid pressure equation and boundary conditions.

For example, tonal suppression is observed in BM and neural responses as well as in psychoacoustics [44] (called masking).

A class of partial differential equation (PDE) ear models and their applications will be discussed. The models are based on both first principles (Newton's laws) and neural phenomenology, and are able to recover known nonlinear phenomena observed in experiments. Model solutions are used to build auditory transforms, especially an orthogonal discrete auditory transform (ODAT), and study sound amplification in hearing aids. Similar in spirit, PDE methods have been successfully developed in image sciences and image processing, [30, 35, 43, 6, 29] among others.

The rest of the paper is organized as follows. In section 2, PDE ear models and their physiological background are introduced. In section 3, auditory transforms built from PDE solutions are discussed. In section 4, numerical methods and results are presented in connection with hearing data in the literature. In section 5, model application to hearing aids gain functions is shown. Concluding remarks are in section 6 .

\section{$2 \quad$ Ear Models}

\subsection{Macromechanics and Passive Models}

The ear modeling ([1, 3, 4, 16, 21, 22, 24, 25, 27, 28, 44] among others) has a long history driven by advancement of experiments ([36, 11, 32, 44] among 
others). The first round of signal processing occurs on the basilar membrane (BM) of the inner ear (cochlea). Figure 1 (left plot) shows a two-dimensional schematic of the cochlear chambers where BM is located, ignoring other connected micro-cellular structures. The cochlear chambers are filled with viscous fluids. The lower cochlea chamber is symmetric with respect to $z=0$. Sound signals generate vibrations on the left boundary $(x, z) \in$ $\{0\} \times[0, H]$, called stapes, which in turn induce fluid motion that goes skew-symmetrically from the upper chamber to the lower chamber through a small hole (helicotrema) at $(L, 0)$. The pressure difference drives the BM into motion. The symmetry allows one to ignore details of helicotrema and concentrate on the upper chamber where the fluid pressure obeys the Laplace equation, along with boundary conditions obtained from balance of mechanical forces.

If the BM is modeled as an elastic spring-mass system, the so called macromechanical system (passive system [27]) is:

$$
\Delta p(x, z, t)=\frac{\partial^{2} p}{\partial x^{2}}+\frac{\partial^{2} p}{\partial z^{2}}=0,(x, z) \in(0, L) \times(0, H), t \in(0, \infty),
$$

where $p(x, z, t)$ is the pressure difference across the BM. The boundary condition on BM is: $\frac{\partial p}{\partial z}(x, 0, t)=2 \rho u_{t t}(x, t), 0 \leq x \leq L$, where $\rho$ is the fluid density, $u(x, t)$ the BM displacement. The upper wall is rigid, so $\frac{\partial p}{\partial z}(x, H, t)=0$, $x \in[0, L], t \geq 0$. The apical wall (the right boundary at $x=L$ ) assumes a zero Dirichlet boundary condition for simplicity, $p(L, z, t)=0$, $0 \leq z \leq H, t \geq 0$. The basal wall (at $x=0$ ) is driven by the input sound $\frac{\partial p}{\partial x}(0, z, t)=2 \rho T_{M} p_{e}(t), 0 \leq z \leq H$, where $p_{e}(t)$ is the input sound pressure at the eardrum, and $T_{M}$ is a bounded linear operator on the space of bounded continuous functions, representing the middle ear filtering. The output depends on the frequency content of $p_{e}(t)$. If $p_{e}=\sum_{j=1}^{J_{m}} A_{j} \exp \left(i \omega_{j} t\right)+$ c.c., a multi-tone input, where c.c. denotes the complex conjugate and $J_{m}$ a positive integer, then $T_{m} p_{e}(t)=\sum_{j=1}^{J_{m}} a_{m}\left(\omega_{j}\right) A_{j} \exp \left(i \omega_{j} t\right)+c . c$, where $a_{m}(\cdot)$ is a functional fit $[10,42,18,20]$ of the experimental data [13] on the filtering characteristics of the middle ear. 
The BM dynamical equation is: $p(x, 0, t)=m u_{t t}+r u_{t}+s(x) u, 0 \leq x \leq$ $L$, where $m$ and $r$ are the BM mass density and damping; $s(x)$ is the BM stiffness function: $s(x)=4 \pi^{2} m(0.456 \exp (4.83(1-x / L))-0.45)^{2}$, based on experimental data $[23,12]$.

For given initial data $\left(u(x, 0), u_{t}(x, 0)\right)$, the passive model is complete as an initial-boundary value problem. Due to the function $s(x)$, the dynamical behavior from a sinusoidal sound (tonal) input is a traveling wave moving in from the left end $x=0$ and settling down as a time periodic standing wave at a corresponding (unique) location on the BM. Hence BM serves as a frequency analyzer of the ear, similar to the Fourier transform. The passive system falls in the category of dispersive PDEs [37].

If one averages the passive system in $z \in[0, H]$, and replaces pressure $p$ by its average, then the Laplace equation simplifies to: $p_{x x}-2 \rho u_{t t}=$ $0, x \in[0, L]$, which together with BM dynamical equation forms the so called transmission line model [34].

\subsection{Micromechanics and Active Models}

The Organ of Corti (Figure 2) rests on the basilar membrane and contains the outer and inner hair cells, which are connected to the tectorial membrane (TM). The outer hair cells (OHCs) are known to contain an active feedback process which amplifies the BM responses for low intensity input, while the inner hair cells transduct mechanical signals to neural electrical impulses to the brain where hearing is finally processed, [11]. Without OHCs, the sensitivity of the ear in vivo disappears [11]. TM also plays a similar role. An active model takes into account such coupling.

A recent micro-mechanical OHC model is the feed-forward (FF) model $[24,25]$. The FF micromechanical model considers the tilt of the OHCs in the longitudinal direction along the cochlear duct, and the $\mathrm{OHC}$ force (denoted by $q(x, t)$ ) on the BM. A balance of forces argument gives the 


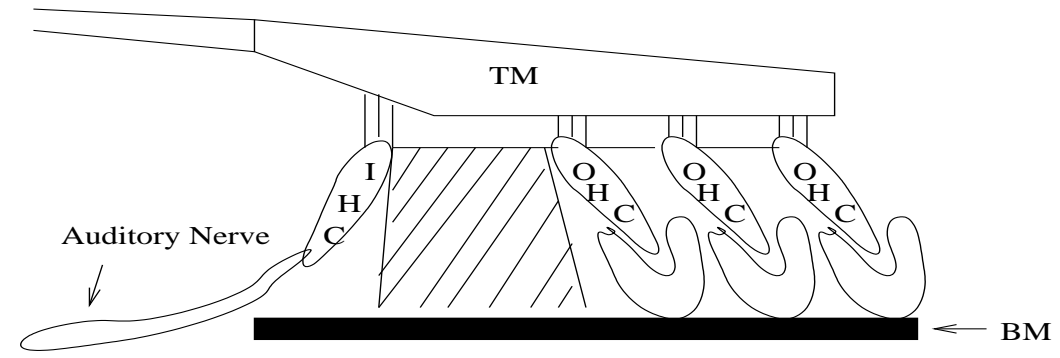

Figure 2: Schematic of Organ of Corti with BM, TM, OHC and IHC.

difference equation $[24,25]$ :

$$
q(x+\Delta, t)=\alpha(x, u, t)(p(x, 0, t)+q(x, t)), \quad x \in[0, L-\Delta],
$$

for some constant $\Delta>0 ; q(x, t)=0$ if $x \in[0, \Delta]$. The function $\alpha$ (the gain factor) is nonnegative and nonlinear such that it is close to a positive constant for $|u|$ small, and decays to zero for large values of $|u|$. A local version is $\alpha_{l o c}(u)=c_{0} /\left(1+c_{2} u^{2}\right), c_{0}, c_{1}$ positive constants.

The larger the $\alpha$, the more enhanced the response is on the BM for the same sound input. Hence by construction, $\alpha$ gives more sensitivity towards lower level sounds in agreement with experiments $[11,32]$. To complete the active system, the BM equation is modified to:

$$
q(x, t)+p(x, 0, t)=m u_{t t}+r u_{t}+s(x) u, \quad 0 \leq x \leq L,
$$

where $m$ and $r$ are positive constants.

To increase regularity of solutions and promote nonlinear interactions on the BM, we proposed the following nonlocal form of the gain factor [18]:

$$
\alpha(x, u, t)=\frac{\gamma}{\sqrt{\lambda \pi}} \int_{0}^{L} \exp \left(-\left(x-x^{\prime}\right)^{2} / \lambda\right) \alpha_{l o c}\left(u\left(x^{\prime}, t\right)\right) d x^{\prime},
$$

where $\gamma$ and $\lambda$ are constants. It turns out that such a nonlocal gain also leads to better stability property of time dependent solutions, and allows us to recover main nonlinear hearing phenomena [18].

The nonlocality of BM nonlinearity is reported in a recent finding of both forward and backward active mechanisms in the cochlea [4], and can also 
be viewed as a phenomenological way of introducing near neighbor neural inhibition $[15,17,8]$. The transmission line models with nonlocal nonlinearities in one space dimension has been utilized for both modeling and sound signal processing $[15,17,8,38,42]$. The two dimensional model offers more accuracy and dynamic range of the amplitudes of solutions.

Another class of coupled model is the resonant TM model [28]. The BM and TM are modeled as two lumped masses coupled by a spring and a damper, with each mass connected to a wall by a spring and a damper. Let $\xi(x, t)=(u(x, t), v(x, t))$ be BM and TM displacement vector, the equations of motion for the passive case are:

$$
\begin{gathered}
M_{\mathrm{p}} \xi_{t t}+C_{\mathrm{p}} \xi_{t}+K_{\mathrm{p}} \xi=F, \\
M_{\mathrm{p}}=\left[\begin{array}{cc}
m_{1} & 0 \\
0 & m_{2}
\end{array}\right], C_{\mathrm{p}}=\left[\begin{array}{cc}
c_{1}+c_{3} & -c_{3} \\
-c_{3} & c_{2}+c_{3}
\end{array}\right], K_{\mathrm{p}}=\left[\begin{array}{cc}
k_{1}+k_{3} & -k_{3} \\
-k_{3} & k_{2}+k_{3}
\end{array}\right]
\end{gathered}
$$

and $F=[p(x, 0, t), 0]^{T}$. The parameters $m_{i}, c_{i}$, and $k_{i}$ are functions of $x$ $[28,20]$. To make the model active, a self-excited force is added to (2.5):

$$
M_{\mathrm{p}} \xi_{t t}+C_{\mathrm{p}} \xi_{t}+K_{\mathrm{p}} \xi=F+F_{\mathrm{a}}
$$

$F_{\mathrm{a}}=\left[\gamma\left[c_{4}\left(u_{t}-v_{t}\right)+k_{4}(u-v)\right], 0\right]^{T}$. The parameter $\gamma \in[0,1]$ is the active gain control, taken as constant in [28]. As in the FF OHC-BM model, we made it into a nonlinear nonlocal functional of BM displacement [20]: $\gamma=\frac{1}{1+\theta N(u)}, N(u)(x, t)=\frac{2}{\sqrt{\lambda \pi}} \int_{0}^{L} \exp \left\{-(x-s)^{2} / \lambda\right\} u^{2}(s, t) d s$, and $\theta, \lambda$ are positive constants.

\section{Auditory Transforms}

\subsection{Invertible Transforms}

In the absence of nonlinearities (e.g. $\gamma, \alpha$ equal to constants), for each single frequency input $e^{i 2 \pi f t}+$ c.c., $f$ frequency, c.c. complex conjugate, the model solutions at large times agree with the exact time harmonic solutions of the form $(P, U, Q)(x, f) e^{i 2 \pi f t}$, where $Q$ denotes the additional coupling variable 
from either $\mathrm{OHC}$ or TM. The $(P, U, Q)$ satisfy elliptic equations in $x$ with complex coefficients subject to Dirichlet-Neumann boundary conditions in the models, see [39] for the analysis on existence and uniqueness in case of the transmission line model. The BM component $U(x, f)$ can be used to build a transform as follows [40]. Let $m^{2}=m^{2}(f)=\int_{0}^{L}|U(x, f)|^{2} d x$. Define the transform on signal $s(t)$ as:

$$
S(t, x)=\int_{R^{1}} d \tau s(\tau) \int_{R^{1}} d f \frac{U(x, f)}{m(f)} e^{2 \pi f i(t-\tau)} .
$$

The function $S(t, x)$ encodes information on (signal) frequency in terms of BM location $x$. The $\tau$ integral is simply Fourier transform denoted by $\hat{s}$, and so the transform can be expressed as: $S(t, x)=\int_{R^{1}} d f \hat{s}(f) \frac{U(x, f)}{m(f)} e^{2 \pi f i t}$, where we see that the amplitude of time harmonic solution modifies (spreads) the Fourier transform $\hat{s}$ in frequency domain. Due to the unit $L^{2}$ norm of the dimensionless function $U(x, f) / m(f)$ in $x$, the transform is invertible in closed form. The transform used our knowledge of ear, and will be called auditory transform. Its discrete version is:

$$
S_{j, m} \equiv \sum_{l=0}^{N-1} s_{l} \sum_{n=0}^{N-1} X_{m, n} e^{i(2 \pi(j-l) n / N)}
$$

where $X_{m, n}$ satisfies $\sum_{m=0}^{M-1}\left|X_{m, n}\right|^{2}=1, \forall n$. Here $M$ is on the order of $N$, and $N$ a power of 2 . The discrete auditory transform (DAT) can be expressed in terms of discrete Fourier transform (DFT) as:

$$
S_{j, m}=\sum_{n=0}^{N-1} \hat{s}_{n} X_{m, n} e^{i(2 \pi n j / N)}
$$

where $\hat{s}_{n}=\sum_{l=0}^{N-1} s_{l} e^{-i(2 \pi l n / N)}$ is the DFT. The DAT inversion formula is:

$$
s_{j}=\frac{1}{N^{2}} \sum_{m=0}^{M-1} \sum_{l=0}^{N-1} S_{l, m} \sum_{n=0}^{N-1} X_{m, n}^{*} e^{i(2 \pi(j-l) n / N)} .
$$




\subsection{Orthogonal Tranforms}

The DAT above is however redundant: $S_{j, m}$ 's are in a space of much higher dimensions $(M \times N>N)$. Redundancy is typical in biological systems. Redundancy means robustness, also lack of efficiency. Is there a way to remove this redundancy while still keeping auditory property in the transform ?

We notice that DFT is recovered from DAT by setting $j=0, M=N$, and $X_{m, n}$ the $N \times N$ identity matrix. In case that $X_{m, n}$ is a nontrivial orthogonal matrix, we still may set $j=0$ to find:

$$
S_{0, m} \equiv S_{m}=\sum_{l=0}^{N-1} s_{l} \sum_{n=0}^{N-1} X_{m, n} e^{-i(2 \pi l n / N)}=\sum_{n=0}^{N-1} X_{m, n} \hat{s}_{n} .
$$

The mapping from $s_{l}$ to $S_{m}$ is orthogonal. The problem reduces to finding an orthogonal matrix $\left(X_{m, n}\right)$ with auditory features. In [41], this is achieved by utilizing the time one map of a discrete nonlocal Schrödinger evolution which conserves the total mass ( $L^{2}$ or Euclidean norm) and smoothes dispersively. The continuum version is:

$$
i u_{t}=\Delta_{x} u+V(x) * u, \quad x \in R^{n}, \quad n \geq 1,
$$

where $*$ is convolution, $V(x)$ is real and even. The conservation of $L^{2}$ norm implies orthogonality, and the auditory property of the ear [33] is incorporated in the potential $V$.

\subsection{Nonlinear Transforms}

The nonlinear transform of a stationary (e.g. quasi-periodic or stationary random) signal $s(t)$ can be defined in terms of the large time asymptotic states of nonlinear PDE models. Let us consider $u(x, t)$ for $t \in[T, T+N d t]$, where $N$ is an integer, $d t$ the sampling time interval determined by a desired sampling frequency $F_{s}$ (e.g. $16 \mathrm{kHz}$ ); $N$ and $T$ are large enough so that a steady (T-independent) Fourier spectrum can be computed from $u(x, T+(1$ : $N) d t$ ) by the $\mathrm{N}$ point DFT. Then we extract the Fourier component at 
the frequency corresponding to the location $x$, use its magnitude to define the nonlinear spectrum. The spectrum can be expressed as a function of frequency by mapping BM location $x$ to frequency. Such a time asymptotic model based spectrum encodes the information on interaction of different frequencies in $s(t)$. If a signal is non-stationary, suitable extension can be done to make it stationary, and nonlinear spectrum can then be computed.

\section{Numerical Methods and Results}

The idea of boundary integral method is used to compute the PDE models $[37,18,20]$. First write pressure $p$ as a boundary integral of $u_{t t}$. Substitution then gives closed equations on $(u, Q)$, where $Q$ denotes the $\mathrm{OHC}$ or TM variable. In case of the FF OHC-BM model, $Q$ can be expressed in terms of $P$ and then in terms of $u_{t t}$. After discretization of the resulting equation in $x$, the semi-discrete equation is: $G_{1}(U) U_{t t}=R U_{t}+S U+G_{2}(U) F$, where $U=\left(u\left(x_{j}, t\right)\right)^{T}$ the BM displacement vector; $x_{j}$ 's are grid points, column vector $F$ depends only on input signal; $M, R$ and $S$ are diagonal matrices with known diagonal entries; $G_{1}$ and $G_{2}$ are matrices nonlinear in $U$. The nonlinear nature of $G_{1}$ and $G_{2}$ comes from the nonlinear nonlocal gain functional $\alpha$ or $\gamma$.

For the time discretization, we use the 2nd order backward differencing. At each time step, one must invert a time dependent mass matrix to advance $U$. Direct inversion is time consuming. Instead, we proceed with an iterative method. The mass matrix can be decomposed into a sum of timedependent and time-independent parts. In the iterative procedure, only the time-independent part is inverted. The spectral radius of the iteration matrix depends on the gain factor $\alpha$ and the time step, and can be made less than one to guarantee fast convergence $[18,20]$. The iterative method is nearly ten times faster than a standard direct solver [18].

The DAT (3.1) and inverse DAT (3.2) are computed with the help of FFT (fast Fourier transform) and inverse FFT. The nonlinear auditory spectrum 
is obtained from taking FFT of nonlinear model solutions over a sequence of sampled times at each discrete location $x_{m}$, then extracting the Fourier component at frequency $f_{m}$ corresponding to $x_{m}$ on BM. The sampled times are such that transients in the solutions are almost died out. In [20], they are later than $15 \mathrm{~ms}$ (mille-second).
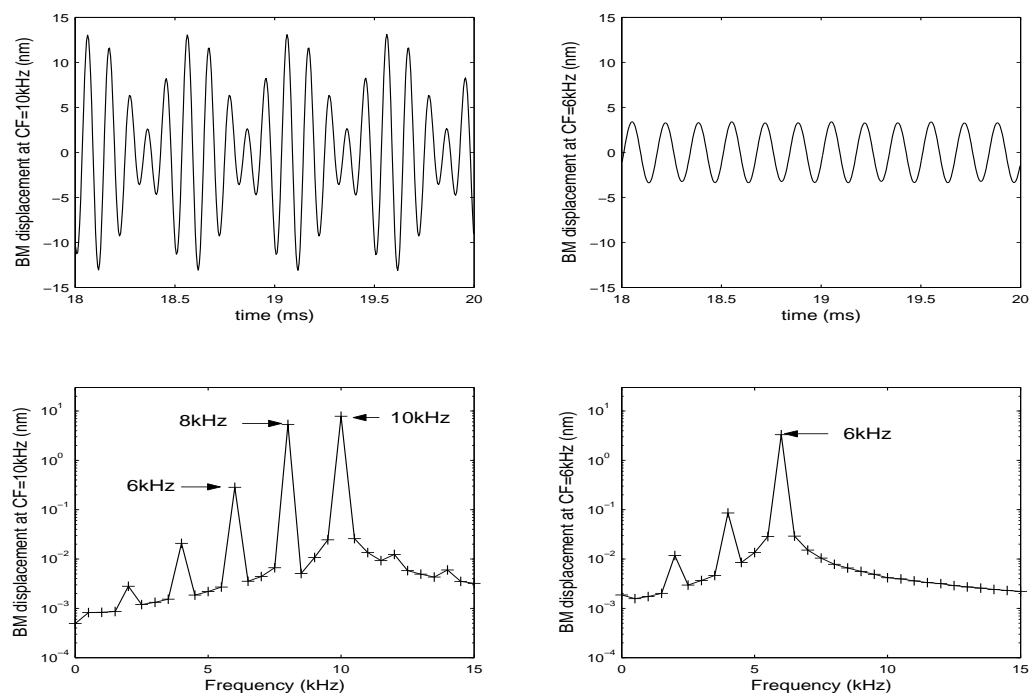

Figure 3: Combination tone $\left(2 f_{1}-f_{2}\right)$ generated from the primary tones $f_{1}=8 \mathrm{kHz}$ (kilo-Hertz) and $f_{2}=10 \mathrm{kHz}$ both at $80 \mathrm{~dB}$ (decibel).

An interesting nonlinear phenomenon is the generation of combination tones (sinusoids) [32]. Figure 3 is computed from the FF OHC-BM model. Two primary tones are at $f_{1}=8 \mathrm{kHz}, f_{2}=10 \mathrm{kHz}$. The top frame shows the $\mathrm{BM}$ displacements in time at $\mathrm{BM}$ locations corresponding to $10 \mathrm{kHz}$ (left) and $6 \mathrm{kHz}$ (right). The bottom presents the FFT-spectra of the timedependent functions on the top. At the $f_{2}=10 \mathrm{kHz}$ place on BM, we see the frequency $6 \mathrm{kHz}\left(=2 f_{1}-f_{2}\right)$ along with $f_{1}, f_{2}$. At the site of $6 \mathrm{kHz}$, a nonlinearly generated oscillation appears and may be perceived by humans. Generation of combination tones is an indication of normal ear function, and is often used in hearing loss detection and hearing tests of infants [26].

The second nonlinear phenomenon is compression, or decrease of sen- 
sitivity (enhancement) towards higher intensity input, as known from experiments [32]. Figure 4 shows the FF OHC-BM model responses at the $\mathrm{BM}$ location corresponding to $10 \mathrm{kHz}$, with input being a click at $x=0$ of duration $0.2 \mathrm{~ms}$ (top frame). A lower intensity click has a higher sensitivity response and longer "ringing" than a higher intensity click. The Figure 4 reaches qualitative agreement with Figure 9 of [32].
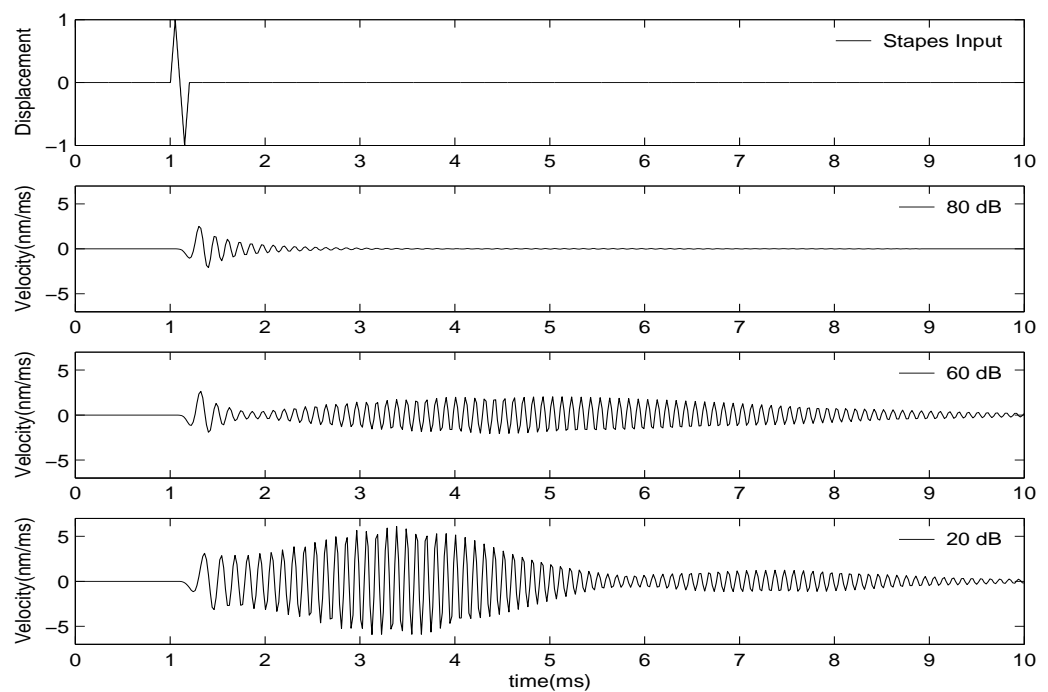

Figure 4: Top panel shows the input click of duration $0.2 \mathrm{~ms}$ at stapes in the time interval of $10 \mathrm{~ms}$. The subsequent plots show the BM velocity normalized by the input intensity level (so called sensitivity) at BM location of frequency $10 \mathrm{kHz}$, at input levels 20, 60 and $80 \mathrm{~dB}$ (decibel).

The third nonlinear phenomenon is suppression among tones or tone and noise. To demonstrate the suppression of noise by tones, Figure 5 shows the nonlinear auditory spectrum computed by the TM-BM model [20]. The input tone is at $2 \mathrm{kHz}$ and $(40,60,80) \mathrm{dB}$ (decibel). The solid line represents noise with tone, the dotted line noise without tone. As tone goes up in intensity, more noise is suppressed from the spectrum. In constrast, suppression is absent in FFT spectrum. 


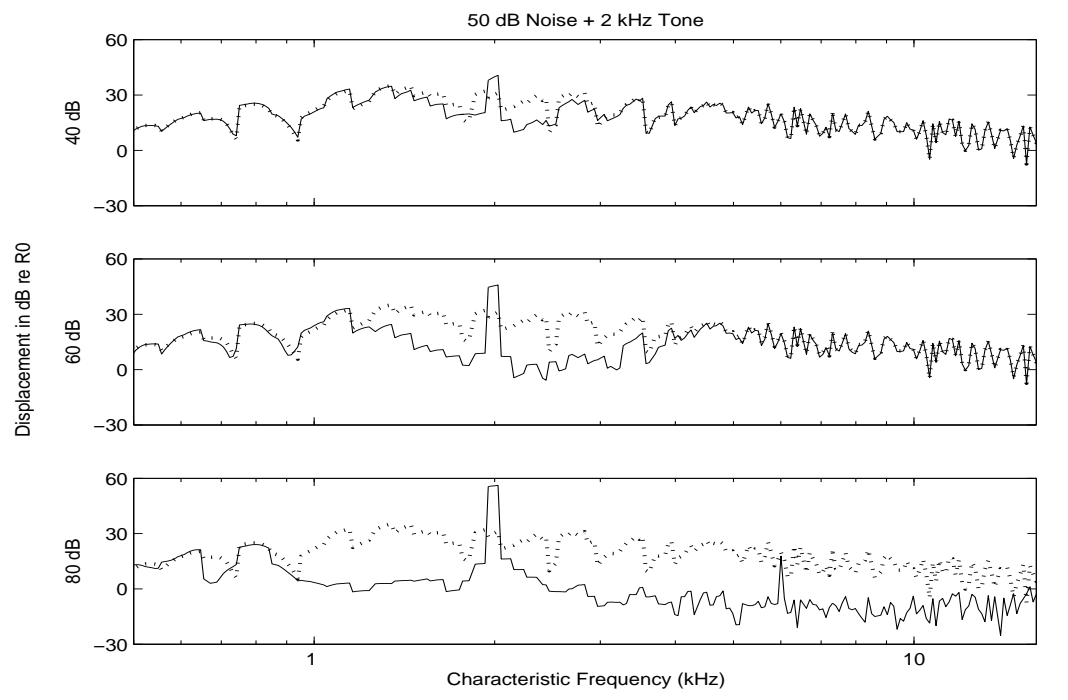

Figure 5: Nonlinear auditory spectrum of input signals consisting of $50 \mathrm{~dB}$ noise and a $2 \mathrm{kHz}$ tone at 40,60, $80 \mathrm{~dB}$ levels. The solid line represents noise with tone, the dotted line noise without tone.

\section{Gain Functions in Hearing Aids}

A common cause of hearing loss is a lack of sensitivity of $\mathrm{OHC}$ due to aging or frequent exposure to loud sounds such as rock music or gunshots [26]. An ear with loss (impaired ear) has raised hearing thresholds and less frequency selectivity. Such an impaired ear can be modeled by our BM-OHC system with active parameter $\gamma$ being small, while a normal ear by a larger $\gamma$. In our study [19], normal and impaired ears will be played by the BM-OHC models with two different values of $\gamma$.

Hearing aids attempt to provide amplification to sounds according to the loss at hearing threshold (audiogram), so that an impaired ear can still hear sounds below its hearing threshold and yet do not feel uncomfortable with loud sounds. The amplification is nonlinear and aims to maximize speech intelligibility (SI). This is the design principle of National Acoustic Laboratories in Australia (NAL), a leading hearing aids research lab. SI is a high 
level quantity that correlates with the percentage correct in understanding of speech, and is empirically defined with the help of experimental data $[7,2]$. The distribution of amplitude gain as a function of frequency will be called gain curves. The NAL-NL1 (a recent NAL product) determines the gain curves by maximizing SI while keeping loudness perception of the impaired ear the same as for a normal ear.

A simplified strategy of computing the gain curves is to find the gain on a pure (single frequency) tone input so that the output from the impaired ear model matches that of the normal ear model. It turns out that certain higher level information has to be used to recover qualitatively the results of NAL-L1 towards high frequency bands. This is because different frequency bands contribute differently to understanding, which is absent in the PDE models. A modified strategy is to provide gain to the input so that the output of impaired ear model matches a suitable percentage of the output of the normal ear or a weighted normal ear output. Such weights can be extracted from the empirical SI functions. Figure 6 shows the comparison with NALNL1. The left column contains three kinds of audiograms (severe, moderate and mild losses) on the two ears being modeled. In the middle column are NAL-NL1 gain functions, and in the right column are gain functions from our method. Qualitative agreement is observed.

\section{Concluding Remarks}

PDE ear models and their applications to sound signal processing offer a fascinating line of research to enhance our ability to utilize sound information. Ear models incorporating multi-level information including perception are especially valuable, and call for further study with the help of hearing data. It is worthwhile to continue to explore auditory transforms with both physiological/psychoacoustic meaning and good mathematical properties allowing for efficient computation. 

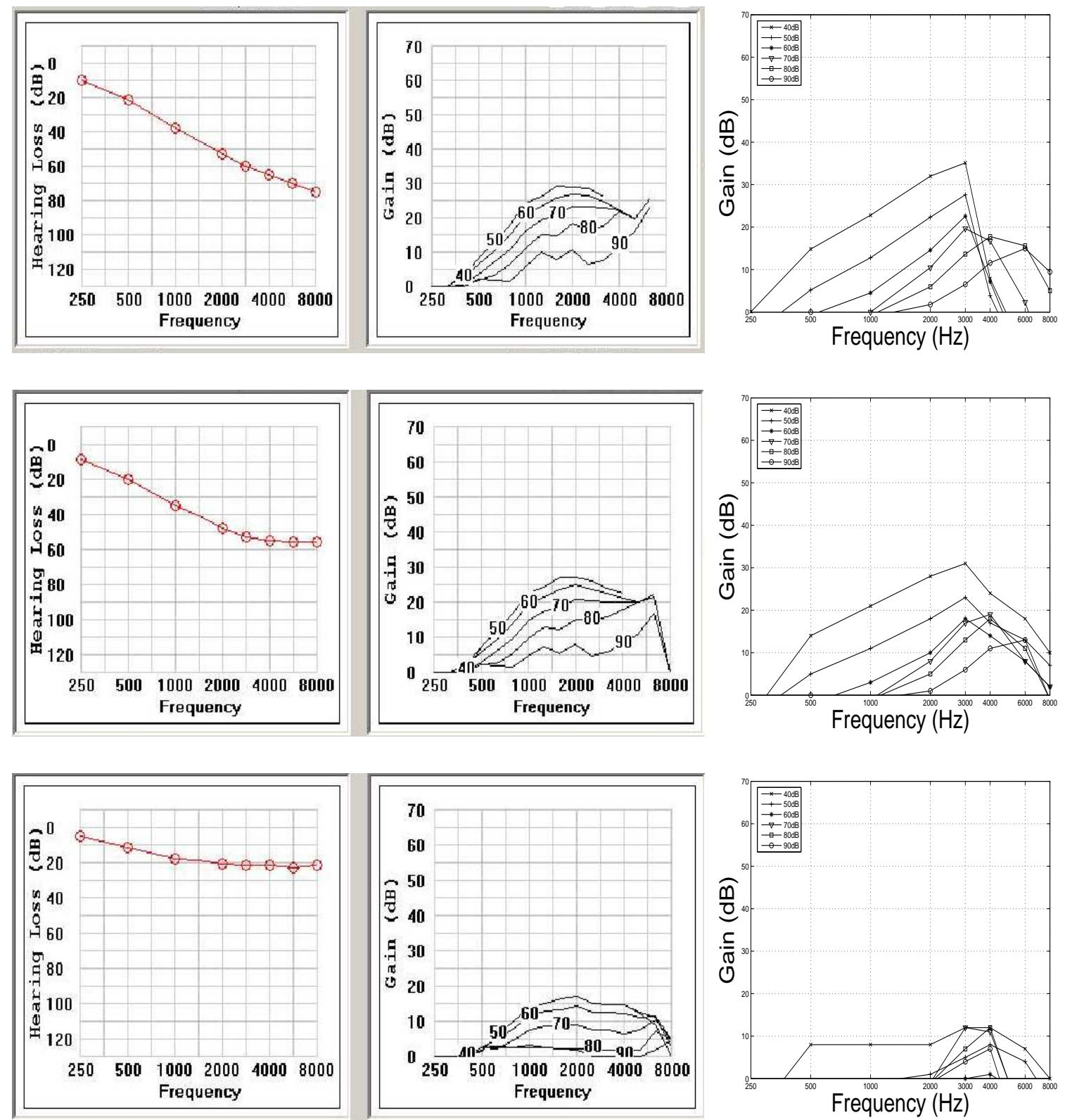

Figure 6: Comparison between the prescription results of NAL-NL1 and our PDE based method [19]. Both methods generate compressive nonlinear gain curves. When the input sound level is moderate (below $60 \mathrm{~dB}$ ), both give the largest gain in the middle frequency region between 2 and $4 \mathrm{kHz}$. 


\section{References}

[1] J. B. Allen, Cochlear Modeling-1980, in: M. Holmes and L. Rubenfeld, eds., Lecture Notes in Biomathematics, Springer-Verlag, 1980, Vol. 43, pp. $1-8$.

[2] ANSI, ANSI S3.5-1997. American National Standard methods for calculation of the Speech Intelligibility Index, New York: American National Standard Institute, Inc.

[3] E. de Boer, Mechanics of the Cochlea: Modeling Efforts, in: P. Dollas, A. Popper and R. Fay, Springer Handbook of Auditory Research, Springer-Verlag, 1996, pp. 258-317.

[4] E. de Boer and A. L. Nuttall, Properties of Amplifying Elements in the Cochlea, in: A. W. Gummer, ed., Biophysics of the Cochlea: From Molecules to Models, Proc. Internat. Symp., Titisee, Germany, 2002.

[5] K. Brandenburg and M. Bosi, Overview of MPEG Audio: Current and Future Standards for Low-Bit-Rate Audio Coding, J. Audio Eng. Soc., (45)(1/2), pp. 4-21 (1997).

[6] T. Chan, "ICCM04 Lecture: Combining PDE and Wavelet Techniques for Image Processing", Hong Kong, Dec, 2004.

[7] T. Ching, H. Dillon, R. Katsch, and D. Byrne, Maximizing Effective Audibility in Hearing Aid Fitting, Ear and Hearing, 22.3:212-224, 2001.

[8] L. Deng, Processing of acoustic signals in a cochlear model incorporating laterally coupled suppressive elements, Neural Networks, 5(1):12-34, 1992.

[9] L. Deng and C. D. Geisler, Responses of auditory-nerve fibers to multiple-tone complexes, J. Acoust. Soc. Amer., Vol. 82, No. 6, 1987, pp. 1989-2000. 
[10] L. Deng and I. Kheirallah, Numerical property and efficient solution of a transmission-line model for basilar membrane wave motions, Signal Processing, Vol. 33, 1993, pp. 269-285.

[11] C. D. Geisler, From Sound to Synapse, Oxford University Press, Oxford, 1998.

[12] D. Greenwood, A cochlear frequency-position function for several species - 29 years later, J. Acousti. Soc. America, 87(1990), pp 25922605.

[13] J. J. Guinan and W. T. Peake, Middle-ear characteristics of anesthesized cats, J. Acoust. Soc. Amer., Vol. 41, No. 5, 1967, pp. 1237-1261.

[14] W. M. Hartmann, "Signals, Sound, and Sensation", Springer, 2000, pp 251-254.

[15] Y. Jau and C. D. Geisler, Results from a cochlear model utilizing longitudinal coupling, in Mechanics of Hearing, Martinus Nijhoff Pub., Delft Univ. Press, 1983, E. de Boer and M. Viergever, pp 169-176.

[16] J.B. Keller and J.C. Neu, Asymptotic analysis of a viscous cochlear model, J. Acousti. Soc. America, 77(6):2107-2110, 1985.

[17] R. Lyon, A computational model of filtering, detection, and compression in the cochlea, IEEE International Conference on Acoustics, Speech and Signal Processing, pp 1282-1285, 1982.

[18] Y-S Kim, J. Xin, A two-dimensional nonlinear nonlocal feed-forward cochlear model and time domain computation of multitone interactions, SIAM J. Multiscale Modeling and Simulation, Vol. 4, No. 2, pp 664-690, 2005 .

[19] Y-S Kim, J. Xin, Y-Y Qi, A Study of Hearing Aid Gain Functions Based on a Feedforward Model of Cochlea, Hearing Research, Volume 215, Issues 1-2, May 2006, pp 84-96. 
[20] M. D. LaMar, J. Xin, Y-Y Qi, Signal processing of acoustic signals in the time domain with an active nonlinear nonlocal cochlear model, Signal Processing, 86(2006), pp 360-374.

[21] R.J. Leveque, C.S. Peskin and P.D. Lax, Solution of a two-dimensional cochlea model using transform techniques, SIAM J. Appl. Math. 45(1985), no. 3, 450-464.

[22] R.J. Leveque, C.S. Peskin and P.D. Lax, Solution of a two-dimensional cochlea model with fluid viscosity, SIAM J. Applied Math, 48(1):191213, 1988.

[23] M.C. Liberman, The cochlear frequency map for the cat: Labeling auditory nerve fibers of known characteristic frequency, J. Acoust. Soc. Amer., 72:1441-1449, 1982.

[24] K.M Lim and C.R. Steele, A three-dimensional nonlinear active cochlear model analyzed by the WKB-numeric method. Hearing Research, 170:190-205, 2002.

[25] K.M Lim and C.R. Steele, Response suppression and transient behavior in a nonlinear active cochlear model with feed-forward. International Journal of Solids and Structures 40:5097-5107, 2003.

[26] B. Moore, "Cochlear Hearing Loss", Whurr Publishers Ltd, London, 2000.

[27] S. T. Neely, Mathematical modeling of cochlear mechanics, J. Acoust. Soc. Amer., Vol. 78, No. 1, 1985, pp. 345-352.

[28] S. T. Neely and D. O. Kim, A model for active elements in cochlear biomechanics, J. Acoust. Soc. Amer., Vol. 79, No. 5, 1986, pp. $1472-$ 1480. 
[29] S. Osher, "ICCM04 Morningside Lecture: An Iterative Regularization Method and Inverse Scale Space for Image Restoration", Hong Kong, Dec, 2004.

[30] S. Osher and L. Rudin, Feature-Oriented Image Enhancement Using Shock Filters, SIAM J. Numer. Analysis, Vol. 27, No. 4, pp 919-940, 1990.

[31] K. Pohlmann, "Principles of Digital Audio", 4th Edition, McGraw-Hill Video/Audio Professional, 2000.

[32] L. Robles and M. A. Ruggero, Mechanics of the mammalian cochlea, Physiological Reviews, Vol. 81, No. 3, July 2001, pp. 1305-1352.

[33] M. R. Schroeder, B. S. Atal and J. L. Hall, Optimizing digital speech coders by exploiting properties of the human ear, Journal Acoust. Soc. America, 66(6), pp 1647-1652 (1979).

[34] M. Sondhi, The Acoustical Inverse Problem for the Cochlea, in: M. Holmes and L. Rubenfeld, eds., Lecture Notes in Biomathematics, Springer-Verlag, 1980, pp. 95-104.

[35] R. Tsai and S. Osher, Level Set Methods and Their Applications in Image Sciences, Comm. in Math Sciences, 1(4), pp 623-656, 2003.

[36] G. von Békésy, Experiments in Hearing, McGraw-Hill, New York, 1960.

[37] J. Xin, Dispersive instability and its minimization in time domain computation of steady state responses of cochlear models, J. Acoust. Soc. Amer., Vol. 115, No. 5, Pt. 1, 2004, pp. 2173-2177.

[38] J. Xin and Y-Y Qi, A PDE based two level model of the masking property of the human ear, Comm. Math. Sciences, Vol. 1, No. 4, 2003, pp. 833840. 
[39] J. Xin and Y-Y Qi, Global well-posedness and multi-tone solutions of a class of nonlinear nonlocal cochlear models in hearing, Nonlinearity, Vol. 17, 2004, pp. 711-728.

[40] J. Xin, Y-Y Qi, An Invertible Discrete Auditory Transform, Communications in Math Sciences, Vol. 3, No. 1, pp 47-56, 2005.

[41] J. Xin, Y-Y Qi An Orthogonal Discrete Auditory Transform, Communications in Math Sciences, Vol. 3, No. 2, pp 251-259, 2005.

[42] J. Xin, Y-Y Qi, and L. Deng, Time domain computation of a nonlinear nonlocal cochlear model with applications to multitone interaction in hearing, Comm. Math. Sciences, Vol. 1, No. 2, 2003, pp. 211-227.

[43] H.K. Zhao, S. Osher, B. Merriman, and M. Kang, Implicit and nonparametric shape reconstruction from unorganized points using a variational level set method. Computer Vision and Image Understanding, 80, pp 295-319, 2000.

[44] E. Zwicker, H. Fastl, Psychoacoustics, Facts and Models, Springer Series in Information Sciences, 22, 2nd edition, New York, 1999. 